\title{
WAVELENGTH VARIATION IN DIRECTIONAL SENSITIVITY OF THE LONG- AND MEDIUM-WAVE SENSITIVE FOVEAL CONES OF RED-GREEN DICHROMATS
}

\author{
F. ZWAS ${ }^{1}$ \\ Vision Research Laboratory. The University of Michigan, Ann Arbor, Michigan 48109. U.S.A.
}

(Receited 19 September 1978)

\begin{abstract}
The intensity of a monochromatic light required just to detect $30 \mathrm{~Hz}$ flicker as the point of entry of the light was changed in $0.5 \mathrm{~mm}$ steps across the eye's entrance pupil and measured throughout the spectrum on 4 protanopes and 5 deuteranopes. In general the directional sensitivity of the foveal cone of these dichromats is minimal in the same part of the spectrum that the spectral sensitivity is maximum. Since the action spectrum of only a single class of cones was evaluated by the procedure, it permits a quantitative comparison of two alternative models for directional sensitivity, one derived from the concept of self-screening, the other from waveguiding. The overall results do not exclude either but are slightly better described by the former than by the latter.
\end{abstract}

\section{INTRODUCIION}

A normal (trichromatic) color match no longer holds when the point of pupil entry of light comprising the mixtures is moved from the center to the edge of the pupil. (Stiles, 1937; Brindley, 1953; Enoch and Stiles, 1961; Alpern, 1978). This is because the action spectrum of at least one, and probably all three of the foveal cones [the absorption of light in the visual pigments of which underlies the match] is different for central than for peripheral pupil entry.

Theories of this color change are of two kinds. One, the self-screening hypothesis" (Stiles, 1937; Brindley, 1953; Walraven and Bouman, 1960; Enoch and Stiles, 1961; Alpern, 1978) assumes that central entry beams are absorbed by pigments in higher concentration than those going through the edge. These macroscopic models rely largely upon concepts developed from geometrical optics. The alternatives emphasize the waveguiding properties of photoreceptors. They relate to the fact that the diameter of individual foveal cones is of the order of the wavelength of light. With this approach the electromagnetic nature of light and the laws of physical optics are more appropriate than geometric optical considerations alone (Enoch, 1960; Wyngaard et al., 1974).

Quantitative confrontation of theoretical expeclations with experimental results are to be found largely for the former variety of theory. While it cannot be doubted that the wave properties of light must play a significant role as light passes down individual photoreceptors, there is no good comparison of expectation from this kind of theory with psychophysical measurements.

This is because until recently the waveguide properties of individual foveal cones could not easily be calculated. While one still cannot from any such theory predict how metameric matches will be upset with pupil entry, Snyder and Pask (1973) have proposed

\footnotetext{
1 Present address: Kresge Eye Institute, Wayne State University, Detroit, MI 48202, U.S.A.
}

a theory of the change in brightness with point of pupil entry (i.e. the Stiles-Crawford effect of the first kind-SCI) based upon electromagnetic analysis of an individual foveal cone which promises this possibility. What is still needed for such a prediction from this theory is knowledge as to how the brightness of the spectrum of monochromatic lights varies with point of pupil entry and with wavelength for each of the three kinds of cones upon which foveal trichromacy depends.

In this and the following paper (Alpern and Zwas, 1979) measurements of the changes in brightness of monochromatic lights with point of pupil entry (when vision depends upon the absorption of light in foveal cones all containing the same visual pigments) are described. In this way, one can derive the change in the action spectrum of each class of cones studied with the change in the point of pupil entry. Coincidentally, data of this kind provide a more realistic test of the Snyder-Pask model than those they used (i.e. the wavelength variation in the brightness with point of pupil entry as perceived by the trichromatic fovea with its three kinds of cone visual pigments).

This paper studies the foveal cones containing a long-wave visual pigment erythrolabe (Rushton, 1965) and those containing chlorolabe (Rushton, 1963) a middle-wave pigment. For isolating these, the action spectrum required just for the detection of $30 \mathrm{~Hz}$ flicker in the foveas of deuteranopic and protanopic dichromats respectively, was measured for different pupil entry points. Rushton (1963, 1965), Alpern. 1974) and Alpern and Wake (1977) have shown densitometrically that these dichromatic foveas are missing respectively a medium-wave or a long-wave sensitive cone pigment; trichromatic foveas similarly evaluated have both a medium and a long wave photolabile pigment. (This observation has been confirmed on the three deuteranopes and the one protanope of the present study for whom it was examined, and there is no doubt of its general validity for the remainder of the subjects as well.) If it could be assumed that the remaining visual pigments in the 
foveas of the subjects were identical to those of a given normal trichromat then the present results, together with those in the following paper (Alpern and $Z$ was, 1979) provide all the information needed to predict the upset of his metameric matches with eccentric pupil entry. Unfortunately, the recent results of Alpern and Pugh (1977) - on deuteranopes-and of Bastian (1976) - on protanopes-show clearly that this assumption is at best only a very crude first approximation. The present results, therefore, fall short of achieving this goal, however useful they may prove in cvaluating the theory of how the color of light changes with change in the point of the pupil it enters the eye (the Stiles-Crawford effect of the second kind).

Careful readers of the preceding paragraph will be curious as to the method used to exclude the dichromatic short-wave sensitive foveal cones. Brindley et al. (1966) and Green (1969) have shown that the cones most sensitive to short wavelength are incapable of detecting flicker in a light pulsing at a frequency larger than $20 \mathrm{~Hz}$. Thus by measuring the action spectrum required for detecting $30 \mathrm{~Hz}$ flicker, the responses evoked in cones all containing a single visual pigment in any given dichromat are studied.

\section{METHODS}

\section{Screening}

The method for diagnosing and recruiting subjects in the laboratory has already been described (Alpern and Wake, 1977). These procedures ensure that all subjects are genuine dichromats, with only one foveal cone visual pigment in the red-green part of the spectrum.

\section{Apparatus}

The two-channel Maxwellian view optical system is shown in Fig. 1. The source $S$ is imaged first by $L_{1}$, then collimated by $L_{2}$ and finally imaged again, this time in the plane of the subject's entrance pupil, by $L_{3}$ (a $155 \mathrm{~mm}$ $f / 2$ Ektar lens). The $1.2^{\circ}$ test and the $8^{\circ}$ surround were provided by the ficld stops $F S_{1} F S_{2}$ mounted in channels 1 and 2 respectively at the primary focal plane of $L_{3}$. The filament images formed in the plane of the pupil by these

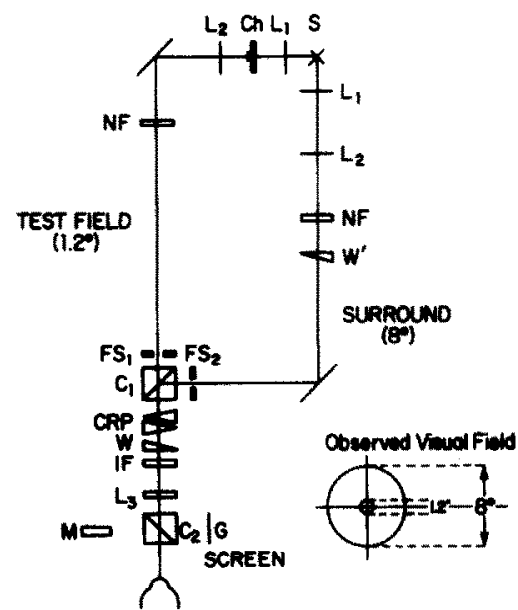

Fig. 1. Optical System. The components of the system are discussed in the text. The insert in the right hand corner shows the spatial configuration of the test and surround fields. two channels were $1 \mathrm{~mm}$ and $0.25 \mathrm{~mm}$ in diameter, $r$ spectively. A chopper $(\mathrm{CH})$ in the plane of the first filament image in the test channel interrupted the light 30 times a second with equal on and off intervals.

The two beams were combined at the mixing cube $C_{1}$. The two superimposed filament images were moved together horizontally across the widely dilated (with 2 drops $1 \%$ mydriacyl) pupil by adjusting the pair of counter-rotating (Risley) prisms (CRP). $F S_{2}$ was mounted on a slide with a 2-dimensional micrometer adjustment. The field stop $F S_{1}$ was an iris diaphragm limiting the test field to $1.2^{\circ}$ but $F S_{2}$ was an annulus, its center a circular area identical to that of $F S_{1}$ being opaque to light.

Neutral and interference filters were all calibrated with a Beckman Spectrophotometer Acta II. Baird-Atomic interference filters $(10 \mathrm{~nm}$ half-bandwidth blocked to infinity on each side of the spectrum) were mounted in the common beam at $I F$. The wedge $W$, a Wratten 96 with a range of approximately $0-3 \log _{10}$ units, was spectrally calibrated with a calibrated Pin-10 photodiode (with the interference filters used in the experiments placed in the optical system)

There were 9 subjects ranging in age between 18-21 years, 4 male protanopes, 4 male and 1 female deuteranope.

The subject's head was positioned by biting into a wax dental bite attached to a drill press table with micrometer adjustments in 3 orthogonal planes. $A$ cube, $C_{2}$. reflected a portion of the light onto a ruled grid. $G$. optically at the same distance from the Maxwellian lens $\left(L_{3}\right)$ as the pupil. The examiner visualized the subject's corneal reflex seen through the microscope, $M$, and adjusted the micrometer screws to bring the subject into alignment. The microscope and grid arrangement were also used to (i) calibrate the translation of the beam across the pupil and (ii) monitor alignment and fixation during the experiment.

After the subject's pupil was widely dilated and he was properly positioned in the bite board, the experimenter adjusted the intensity of the surround with $W^{*}$ until it matched the brightness and color of the center, the intensity of the latter being just above the fusion point of flicker. All subsequent intensity adjustments were made by the subject with the wedge $W$ varying center and surround together. An important feature of the method is that the adaptation state of the retinal regions viewing the central test and its surround were always identical.

The SCI was obtained by having subjects determine the minimum amount of light needed just to detect the flicker of the central test field at $30 \mathrm{~Hz}$, as a function of pupil position. This was done in $0.5 \mathrm{~mm}$ steps along the horizon. tal meridian of the subject's widely dilated pupil. At each point of entry 3-4 readings were obtained and averaged. The guiding principle of this technique is that only the long-wave or the medium-wave cone mechanism follow the fickering stimulus. Since the curve that plots critical fusion frequency $(C F F)$, as a function of log intensity, rises steeply at $30 \mathrm{~Hz}$, the minimum luminance needed just to detect flicker at $30 \mathrm{~Hz}$ for each entry point provides a sensitive fixed criterion. Coble and Rushton (1970) have documented the validity of this technique for the measurement of the SCI effect objectively by retinal densitometry. The same procedure was followed with a variety of monochromatic wavelengths throughout the spectrum. Almost always, two wavelengths were studied on the same day. with suitable rest intervals between measurements as required; occasionally only a single wavelength could be tested on a given day.

\section{RESULTS}

Stiles (1937) found that $I_{r}(\lambda)$ the photons $\mathrm{sec}^{-1} \mathrm{deg}^{-2}$ of visual angle required in a monochromatic light to reach some fixed brightness criterion 
at different points of pupil entry $r$ (in millimeters of distance from the geometric center of the pupil) could be described to very good approximation with the empirical equation

$$
\log \eta=\log \frac{I_{r_{m}}(\lambda)}{I_{r}(\lambda)}=-\rho(\lambda)\left(r-r_{m}\right)^{2}
$$

in which $\rho(\lambda)$ is a parameter which characterizes the width of the curve, and $r_{m}$ represents the distance from the geometric center of the pupil to the point that requires the least light $I_{r_{m}}(\lambda)$ to achieve the criterion. This was the equation of choice not only because it provided a better description of the results overall than the alternative (i.e. a Gaussian curve) but because its use facilitates a quantitative comparison of the data with the empirical and theoretical results of Stiles, Walraven and Bouman and Snyder and Pask.

In Fig. 2 representative results for one protanope (left) and one deuteranope (right) are given for 10 wavelengths throughout the spectrum. Each graph is a plot of

$$
\log \frac{I_{r_{m}}(\lambda)}{I_{r}(\lambda)}
$$

as a function of point of pupil entry $r$, the individual points being the average of the determinations obtained in a single session. The smooth curve drawn through the experimental points is the best fitting par- abola defining Stiles' equation as obtained from a computer minimization of the squares of the (vertical) deviations. (Although no obvious example occurs in Fig. 2, occasionally at one or two pupil entry points near one edge, measurements were distinctly more sensitive than expected from a parabola defined by the remaining measurements in the run. Whenever this occurred, such points were not included among the points which the computer used to determine the best fitting parabola.) Since these curves described the measurements with precision, as the "typical" results illustrated in Fig. 2 attest the values of $\rho(\lambda), r_{m}(\lambda)$ and $I_{r}(\lambda)$ obtained from these computer solutions were then used in further analysis.

\section{A. $r_{m}(\lambda)$}

As the typical results illustrated in Fig. 2 emphasize, there was no dramatic or consistent change in $r_{m}$ with wavelength. Similar results were obtained for cach dichromat and nothing in the individual results, nor their respective averages, suggested that longwave sensitive visual pigments of deuteranopes are found in cells pointing to a different part of the pupil than those containing medium-wave visual pigments of protanopes. This negative result is hardly surprising, but it is noteworthy in view of the finding in the next paper (Alpern and Zwas, 1979) which suggests that in one deuteranope the long-wave sensitive cones may well be pointing toward a different part of the pupil than the short-wave sensitive cones.

T.N. (DEUTERANOPE)

J.S. (PROTANOPE)

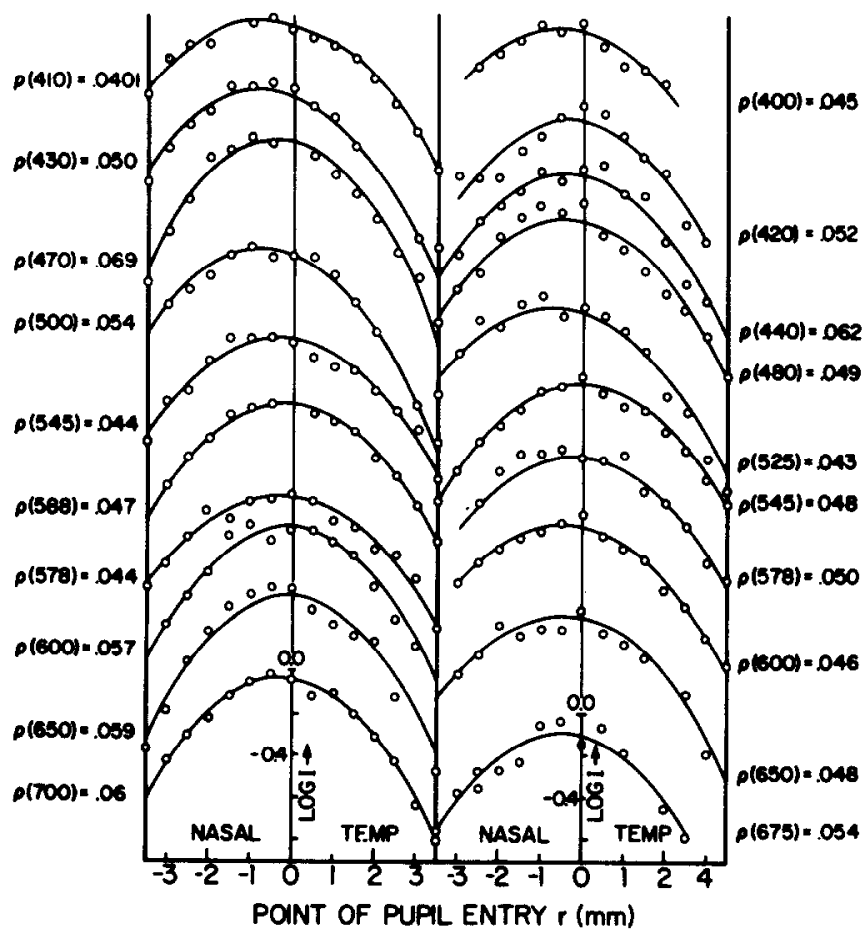

Fig. 2. Illustrates the Stiles-Crawford effect of the first kind for a deuteranope and a protanope for 10 different wavelengths. The individual curves show the change in minimum intensity required to detect $30 \mathrm{~Hz}$ flicker for each point of pupil entry. Each point represents the mean of three measurements obtained in a single session and is plotted as a function of pupil position. The continuous curve is a parabola. It is fit to the data by minimizing the squares of the vertical deviations. The parameter $\rho(\lambda)$ which is a measure of directional sensitivity is obtained from the parabolic equations. 


\section{B. $I_{r}(\lambda)$ action spectrum through different points of pupil} entry

The way $I$, changed with wavelength is plotted for two values of $r$, namely $r_{m}$ (circles connected by a broken line) and $r-r_{m}=3.5 \mathrm{~mm}$ (continuous line), are shown for each of the 4 protanopes (Fig. 4) and 5 deuteranopes (Fig. 3). In Fig. 2 the computer solutions were plotted directly, but in Figs 3 and 4 their values have been corrected for losses in the eye media before plotting. Hence the two latter figures illustrate action spectra at the retina. Said and Weale (1959) measurements of lens density as a function of wavelength for a 21-year-old subject were used. The calculation also allowed for the fact that light entering at the edge of the pupil passes through a smaller thickness of lens than that entering at the pupil center Mellerio's (1971) results were used to make this correction. Finally, the absorption spectrum of the macular pigment was assumed to be that given in Table 2.8 p. 219 of Wyszecki and Stiles (1967). All these corrections are only approximations--not one of them comes from the eyes to which they have been applied but they are useful in making a reasonable guess, for only spectra of this kind are appropriate for testing theories of the upset of metameric matches with change in the point of pupil entry of the light

To facilitate the comparison of the action spectra at $r_{m}$ with that obtained at $r=3.5 \mathrm{~mm}$, the latter has

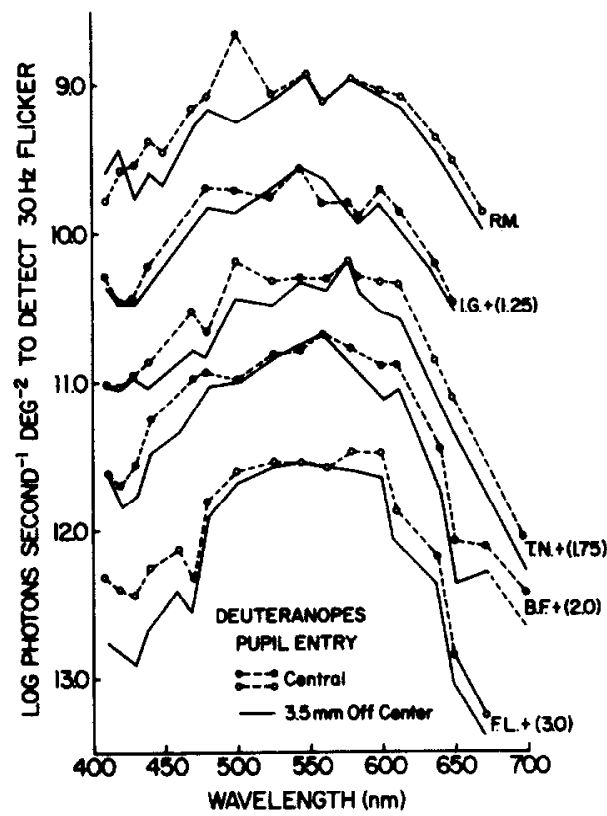

Fig. 3. The change in action spectra of the long-wave mechanism of 5 deuteranopes on changes from central pupil entry to a point $3.5 \mathrm{~mm}$ away is shown. The individual points are obtained by measuring the minimum intensity required to detect $30 \mathrm{~Hz}$ flicker. The broken lines connecting the open and solid circles represent the action spectra for central pupil entry after correction for losses in the eye media. The number on the side of each curve represents the shift in log units from the top curve. The solid curves immediately beneath the dotted curves represent the respective $(r=3.5 \mathrm{~mm})$ edge pupil action spectra corrected for eye media. These curves were shifted by eye to correspond to the maximum sensitivity of each particular subject.

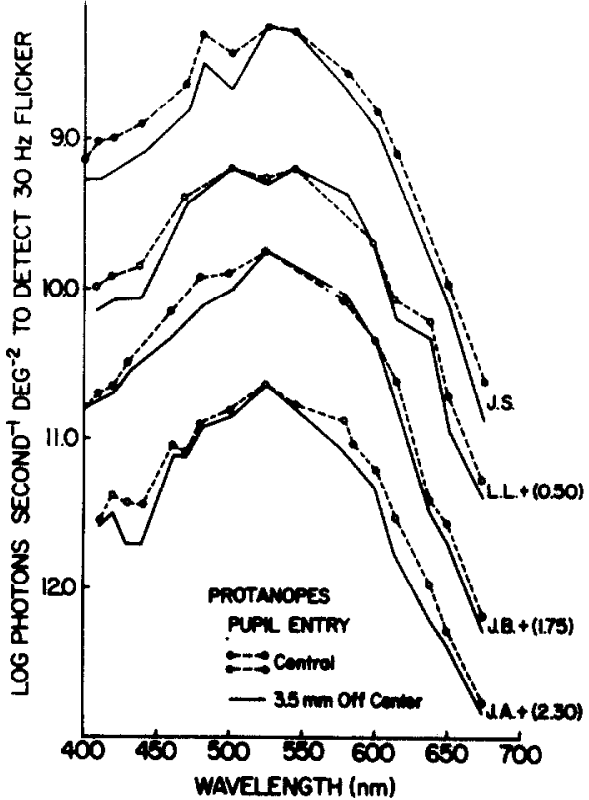

Fig. 4. The change in action spectra of the medium-wave cone mechanism of 4 protanopic foveas on change from central pupil entry to a point $3.5 \mathrm{~mm}$ away is shown. The individual points are obtained by measuring the minimum intensity required to detect $30 \mathrm{~Hz}$ flicker. The broken lines connecting the open and solid circles represent the action spectra for central pupil entry after correction for losses in the eye media. The number on the side of each curve gives the amount of the shift in log units from the top curve. The solid curves represent the respective $(r=3.5 \mathrm{~mm})$ edge pupil action spectra corrected for eye media. These curves were shifted by eye to correspond to

the maximum sensitivity of each particular subject.

been vertically shifted to coincide with the former in these two figures at the wavelength of peak spectral sensitivity. [The amount of this shift required can be readily calculated from the relevant value of $\rho\left(\lambda_{\max }\right)$ in Fig. 5 (cf. below).]

It is noteworthy that in each case the action spectrum for the mechanism absorbing the light which passes through the center of the pupil is considerably broader than that absorbing the light entering through a point close to its edge. This is qualitatively the result expected for the theory of self-screening. To evaluate such theories more quantitatively, it is useful to study $\rho(\lambda)$.

\section{Wavelength variation of $\rho(\lambda)$}

The values for $\rho(\lambda)$ corrected in the way outlined in section (B) above are plotted as a function of wavelength for the 4 protanopes on the left and the 5 deuteranopes on the right of Fig. 5. The vertical line through each plotted point is limited by the standard deviation of the least square estimate of $\rho(\lambda)$ obtained from the computer fit to the Stiles parabola.

These data can be compared with other attempts to determine how the brightness directional sensitivity of the long- and medium-wave sensitive cones varies with wavelength. Two such estimates have been made. Stiles (1939) made estimates from two color increment thresholds on his own eye as to how the test directional sensitivity varied with wavelength for the long- 


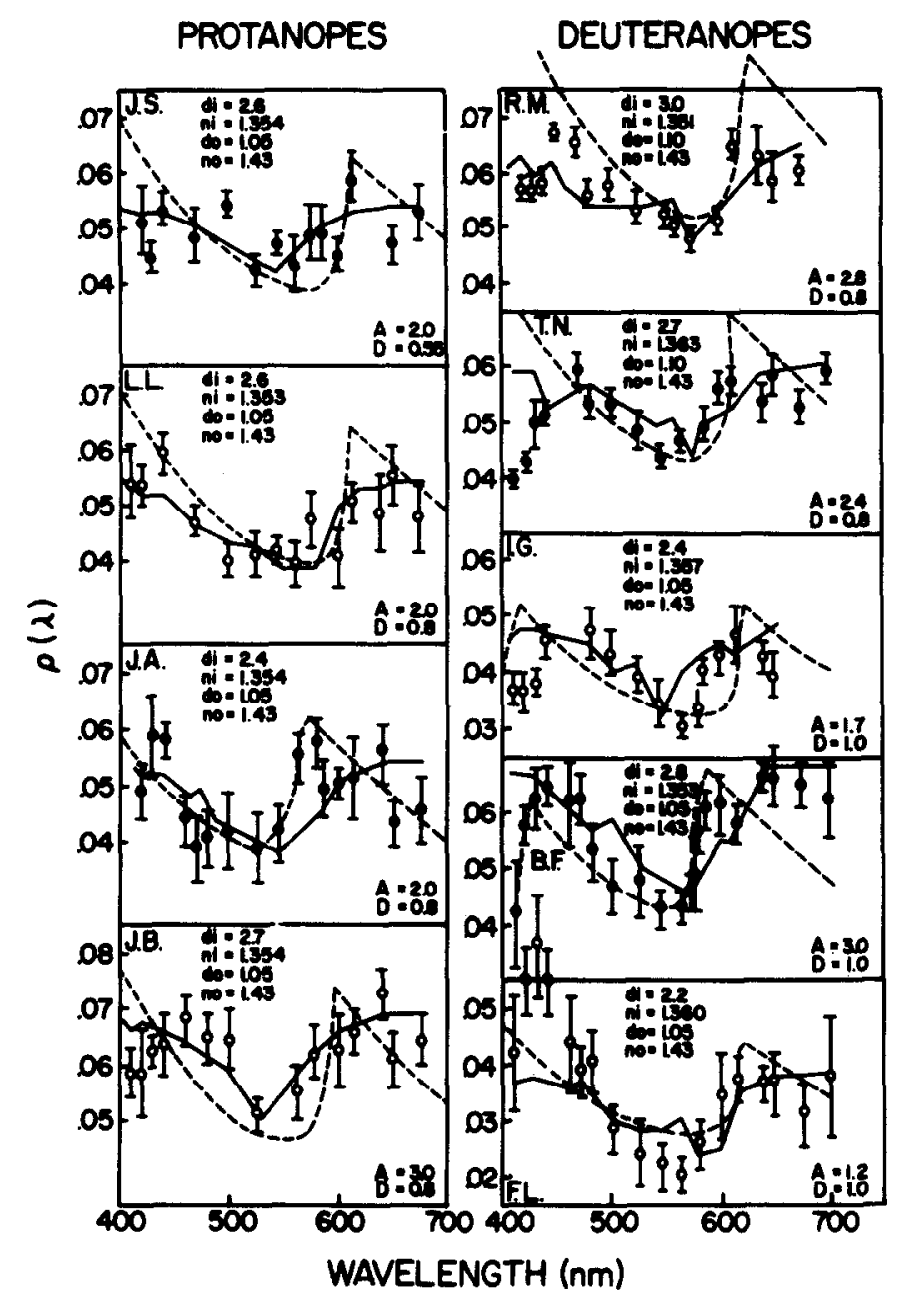

Fig. 5. The directional sensitivity parameter $\rho(\lambda)$ as a function of wavelength for 5 deuteranopes and 4 protanopes. The open and solid circles represent the computed direction sensitivity for each subject. The continuous lines represent the computed directional sensitivity based on the self-screening hypothesis using the action spectrum of each subject. The parameters $D$ and $A$ were varied to obtain the best fit to the data. The dashed lines represent the computed curve based on the waveguide model. The parameters $d_{i}, n_{i}$ and $d_{o}$ were varied to obtain the best fit to the data.

and medium-wave sensitive mechanism. Enoch and Stiles (1961) used the measurements of both kinds of Stiles-Crawford effects on one normal trichromat (Enoch) to infer how $\rho(\lambda)$ would vary with $\lambda$ for each cone mechanism first guessing the action spectrum of each cone mechanism for central entry of the light. There is reasonable agreement between these separate estimates insofar as the long-wave sensitive cones are concerned but the two procedures gave widely different relations for $\rho(\lambda)$ for the medium-wave sensitive cones. The results in Fig. 5 follow closely the estimates of Enoch and Stiles (1961). Indeed, shifting their values (for medium-wave cones) up by a constant amount, 0.015 , yields a good description of the average of results of all the protanopes in Fig. 5. A similar maneuver with Enoch and Stiles (1961) estimate as to how directional sensitivity of the longwave sensitive cones of Enoch's eye varies with wavelength provides a good description of the average results of the 5 deuteranopes in Fig. 5. This agreement is hardly fortuitous. On the other hand, it may not prove as fundamental as it appears at first glance because the action spectra of light entering the center of the pupil used for calculating these values were "Koenig fundamentals" essentially obtained by assuming protanopia and deuteranopia to be reduced forms of normal trichromacy.

In evaluating the relative merits of alternative models of directional sensitivity the explicit model of Walraven and Bouman (1960) as modified by Enoch and Stiles (1961) and that of Snyder and Pask (1973) have been applied to the results shown in Fig. 5. In the Appendix a short summary of the equations used in the calculations of directional sensitivity for these models is presented. No attempt is made to duplicate the theoretical development found in the original papers to which readers are referred for explicit details.

\section{Self-screening}

In the theory of self-screening there is a wavelength independent loss of light to the surrounding tissue which increases with increasing obliquity and is zero for normally incident light. For given values of leak- 
age density $A$ and optical density of the photosensitive pigment $D$ at the $\lambda_{\max }$, the form of $\rho(\lambda)$ is uniquely determined.

Enoch and Stiles' (1961) methods for determining $D$ and $A$ were used. A computer program generated a family of curves of $\rho$ as a function of $\lambda$ by using equations (5) and (6) of the appendix. That optimal combination of these parameters was used to obtain the best fit to the experimentally measured values of $\rho(\lambda)$ which are plotted in Fig. 5. The results of this curve fitting procedure are shown as the continuous lines for each of the 9 dichromats of this study in the same figure. As a first order approximation agreement is reasonable, although in at least half of the subjects the measured directional sensitivity is systematically smaller at the short-wave spectral extremity than this theory leads one to expect.

\section{E. Waveguide model}

In the model of the Stiles-Crawford brightness effect of Snyder and Pask (1973) the variation of the magnitude of the effect with wavelength is attributed to diffraction and interference associated with dielectric waveguide-model propagation in the cones. In this formulation the directional sensitivity of the receptor is quite independent of the spectral properties of the visual pigment in the cone outer segments, and in this respect the theory differs sharply from the "self-screening" hypothesis evaluated above. The directional properties of a single cone serves to model the psychophysically measured directional sensitivity which is determined by a large number of cones in the $1.2^{\circ}$ test field used in this study. In this model the directional properties are determined by summing the total modal power which can be absorbed by the visual pigment in the cone outer segment (that is, the modal power at the entrance to the taper which is assumed to be sustained by the outer segment structure). All modes which are excited in the inner segment but are not capable of being sustained by the outer segment structure arc assumed lost to the surrounding medium. The directional sensitivity of a single long- (or a medium-) wave sensitive cone is evaluated by using equations (7) and (8) given in the Appendix. These equations were used to generate a large family of curves for $\rho(\lambda)$ as a function of $\lambda$ by varying the parameters for the index of refraction of the inner segment $n_{i}$, the diameter of the inner segment $d_{i}$ and the diameter of the outer segment $d_{o}$ The index of refraction for the outer segment and the surrounding medium were assumed to be equal to $n_{0}=1.43$ and $n_{s}=1.34$, respectively. [The latter is the value used by Snyder and Pask (1973) while the former is the midpoint of the range they examined. Since from equation 9 it is clear that the effect of varying $n_{o}$ is the same as varying $d_{o}$, without a good experimental way of making the appropriate choice, it was decided to hold the former fixed and vary the latter, in an analysis which can at best only approximate the actual relationships.] The one member of this family of curves judged by eye to approximate most closely the experimental measurements was selected for each dichromat. The results are plotted on the individual graphs of Fig. 5 as dotted smooth curves. The optimum values for the par- ameters $n_{i}, d_{i}$ and $d_{o}$ which yielded these curves are also given in each case.

\section{DISCUSSION}

Insofar as the measurements in Fig. 5 are concerned. the results for deuteranopes are in agreement with expectation of the directional sensitivity of the longwave sensitive cones derived from color matching (Enoch and Stiles, 1961) which, in turn, are similar in a general way to the directional sensitivity of what is now known as $\pi_{5}(\lambda)$ obtained by Stiles (1939) for $\lambda \geq 500 \mathrm{~nm}$. Enoch and Stiles (1961) determination of the directional sensitivity of the medium-wave sensitive cones from color matching are inconsistent with the estimate of how the directional sensitivity of what is now called $\pi_{4}(\lambda)$ varies with wavelengths (Stiles, 1939). The protanope results in Fig. 5 are in much better agreement with expectation from color matching than from the increment threshold data.

There is a suggestion in almost all such measurements both in this and the above cited papers for the long- and medium-wave sensitive cones to be less directionally sensitive at short-wave spectral extreme than in the violet near $450 \mathrm{~nm}$. This tendency where it occurs is not predicted by the self-screening theory. Waveguide theory leads to the expectation of some trend of this kind for $\lambda \leq 410 \mathrm{~nm}$ but in Fig. 5 the addition of this third mode in practice makes no substantial contribution toward a better quantitative description of any of the data. These discrepancies may be attributed in part to inaccurate estimates of light losses in the lens. The Said and Weale (1959) data used may perhaps be a conservative estimate. They were selected because they represent the only values directly measured in vivo which cover the spectrum over a sufficient range and for subjects of the proper age. The alternatives are either based on in vitro estimates which may well reflect post mortem artifacts or be deduced from indirect psychophysical estimates using assumptions which make the inferred absorption spectrum less compelling. It seems unlikely that uncertainties of this kind can deal with the pronounced fall in directional sensitivity found in shortwave extreme in some dichromats (for example, deuteranopes TN, BF and protanopes JB and JA). To the extent that this is the case, those measurements represent a genuine difficulty for either of the two theoretical interpretations analyzed here.

There is no obvious clustering of the best fitting parameters for either theory which separates results for protanopes from those for deuteranopes. This may only mean that whatever is responsible for the changes in the action spectra of long- and mediumwave sensitive cones with pupil entry is a feature of the receptors themselves rather than the pigment they contain. [Adopting the view (Helmholtz, 1896; Alpern and Moeller, 1977) that a pigment is missing from the retina of these dichromats not because the cells which normally would contain them are also missing. but because these cells are filled with one, or the other, of the two remaining cone pigments.] Accepting the idea that receptor, as distinct from pigment. characteristics are responsible for the action spectrum change of a given receptor species with change in pupil entry is tantamount to rejecting the self-screen- 
ing explanation of the change in action spectrum with change in point of pupil entry for the long- and medium-wave sensitive cones. The results of this study together with other available evidence suggest that adopting such a position in favor of the viable quantitative alternatives at the moment is clearly premature.

The self-screening hypothesis provides, in fact, at least as satisfactory a quantitative explanation for the wavelength variation of the directional sensitivity results in Fig. 5. as does the Snyder-Pask waveguide theory. Self-screening predicts that the wavclength of maximum spectral sensitivity is precisely the wavelength of minimum directional sensitivity. A trend of this kind is evident in all of these results; however, the flicker thresholds are so sensitive to prior retinal excitation by pulsing lights (Alpern and Sugiyama, 1961) that the resulting spectra (in Figs 3 and 4) are imprecise. Although the practice of pooling measurements from different dichromats of the same class to determine an average curve is not without methodological difficulty (Alpern and Pugh, 1977) this approach has been followed in Fig. 6 in order to obviate as far as possible irregularities of this kind in individual flicker measurements. In evaluating these results it is clear that for the medium-wave sensitive cones of protanopes the average spectral sensitivity curve peaks at $525 \mathrm{~nm}$ which is precisely the wavelength of the minimum in the average directional sensitivity curve in excellent agreement with selfscreening. On the other hand for the long-wave sensitive cones of the average deuteranopes these values

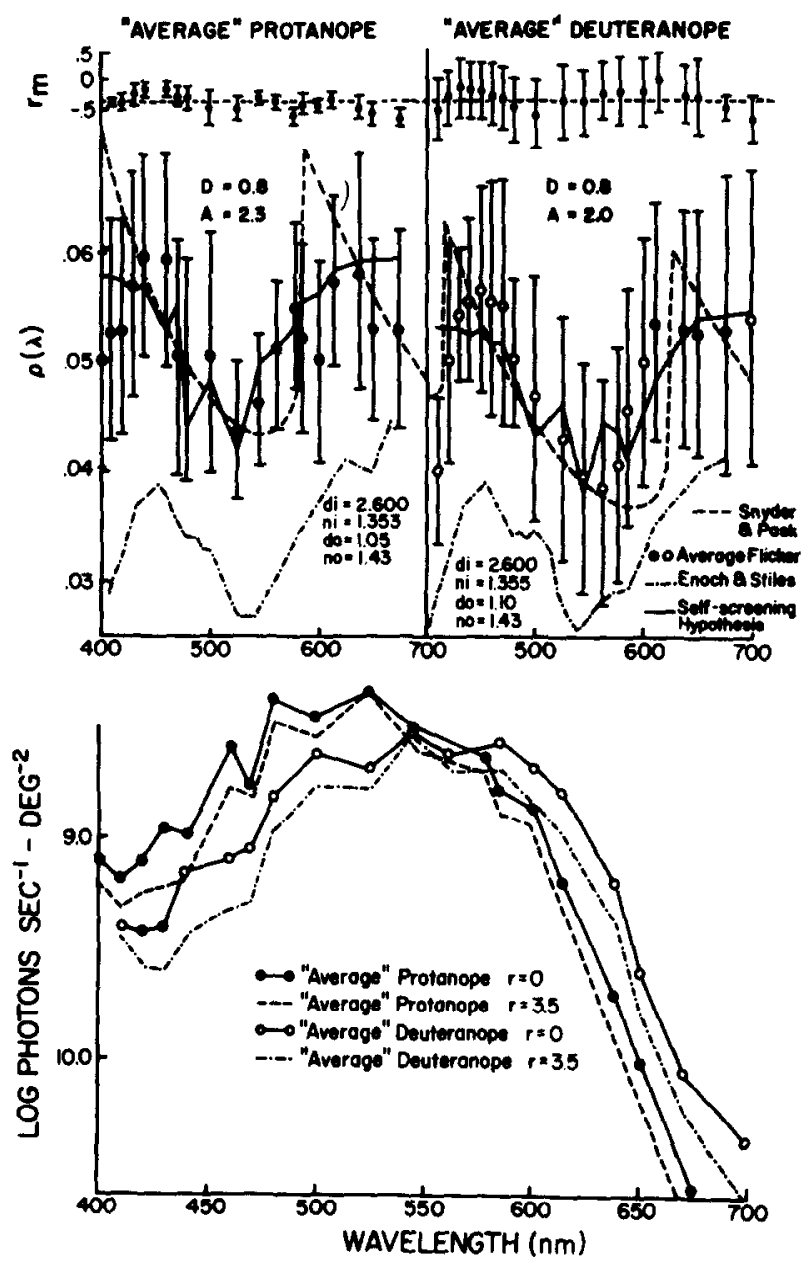

Fig. 6. The directional sensitivity parameter $\rho(\lambda)$ as a function of $\lambda$ is plotted for an "average" protanope (solid circles) and an "average" deuteranope (open circles). The bars through the points represent the standard deviation. The continuous lines give the computed directional sensitivity based on the self-screening hypothesis using the averaged action spectrum for each group of dichromats. The best fitting parameter $D, A, d_{i}, n_{i}$ and $d_{o}$ are also shown. The dash point curve are the results of Enoch and Stiles (1961). On the top of the figure the average $r_{m}$ is plotted for the deuteranopes and protanopes with the bars representing the standard deviation. At the bottom of the figure, the change in action spectrum for the average of 4 protanopes and 5 deuteranopes is presented. The lines connecting the solid and open circles represent the average action spectrum for central pupil entry after correcting for the eye media. The broken curves immediately beneath the continuous ones represent the respective $(r=3.5 \mathrm{~mm})$ edge pupil spectra. These curves were shifted by eye to correspond to the maximum sensitivity of each particular subject. 
$545 \mathrm{~nm}$ and $560 \mathrm{~nm}$ respectively are slightly disparatc but not unreasonably so given the rather prominant irregularities which persist even in the average curve.

While in general all of these observations are consistent with self-screening the overall pigment densities one needs in order to explain the results quantitatively $(0.8-1.0$ common logarithmic units) is rather large. Assuming that the recent microspectrophotometry measurements (Bowmaker et al. 1978) of specific density of chlorolabe in monkey green cones $\left(0.015 \pm 0.004 \mu \mathrm{M}^{-1}\right)$ one calculates an outer segment length of $53 \mu \mathrm{M}$ for medium-wave sensitive foveal cones of protanopes JB, JA and LL and a length of $37 \mu \mathrm{M}$ for protanope JS. On the other hand using Bowmaker et al. value of $0.013 \pm 0.002 \mu \mathrm{M}^{-1}$ for the specific density of erythrolabe leads to a calculated length of $77 \mu \mathrm{M}$ for the length of the long-wave foveal cones of deuteranopes $F L, B F$ and $I G$ and a value of $62 \mu \mathrm{M}$ for deuteranopes TN and RM. These values are somewhat larger than expected from histology (36- $40 \mu \mathrm{M}$ for man; 42-67 $\mu \mathrm{M}$ for monkey) (Polyak, 1941 ; Dowling, 1965) but perhaps not unreasonably so given inaccurate shrinkage corrections and individual differences.

In balance, quantitative comparison of these models of the wavelength variation of the brightness directional effect for the long- and medium-wave sensitive cones of the "average" dichromats in Fig. 6 would probably decide in favor of self-screening. But even those results can not categorically exclude the Snyder-Pask alternative and the matter must remain open. Perhaps the truth falls somewhere between. A specific model of this kind (Wyngaard et al.. 1974) does not deal better with the present results but, no doubt, others are possible. Indeed, the quantitative agreement between either theory and measurement is so approximate that it would be a mistake to interpret the current state of understanding sufficient to abandon an imaginative search for other possibilities insofar as long and medium-wave sensitive foveal cones of dichromats are concerned. That this is certainly true for the short-wave sensitive contes of dichromats, the reader may convince himself by examining the evidence of the following paper (Alpern and Zwas, 1979)

Acknowledgements - I am very grateful to Professor Mathew Alpern for the invaluable help and guidance he has provided in this work. The work on this project was supported by Grant No. EY-00197 to M. Alpern from the National Eye Institute and by an NEI postdoctoral fellowship (EY-505011), by a training grant for vision research to the University of Michigan EY-07022 and by a postdoctoral research fellowship (F-257) from Fight for Sight, Inc (New York)

\section{REFERENCES}

Alpern M. (1974) What is it that confines in a world without color? Invest. Ophthal. 13, 647-674.

Alpern M. (1978) Lack of uniformity in color matching. In preparation.

Alpern M. and Moeller J. (1977) The red and green cone visual pigments of deuteranomalous trichromacy. $J$. Physiol. 266, 647-675.

Alpern M. and Pugh E. M. Jr (1977) Variation in the action spectrum of erythrolabe among deuteranopes. $J$. Physiol. 266, 613-646.

Alpern M. and Sugiyama S. (1961) Photic driving of the critical flicker frequency. J. opt. Soc. Am. 51, 1379-1385.
Alpern M. and Wake T. (1977) Cone pigment in human deutan color vision defects. J. Physiol. 266, 595-612.

Alpern M. and $Z$ was F. (1979) The wavelength variation of the directional sensitivity of the Stiles $\pi_{1}(\mu)$. This issue, p. 1077.

Bastian B. L. (1976) Individual differences among the photopigments of protan observers. Dissertation. University of Michigan.

Bowmaker J. K., Dartnall H. J. A., Lythgoe J. N. \& MolIon J. D. (1978) The visual pigments of rods and cones in the Rhesus monkey Mucaca mulatta. J. Physiol. 274. 329-348.

Brindley G. S. (1953) The effects on colour vision of adaptation to very bright lights. J. Physiol. 122. 332-350.

Brindley G. S.. Du Croz J. J. and Rushton W. A. H. (1966) The flicker fusion frequency of the blue sensitive mechanism of colour vision. J. Physiol. 183, 497-500.

Coble J. R. and Rushton W. A. H. (1971) Stiles-Crawford effect and the bleaching of cone pigments. $f$. Physiol. 217, 231-242.

Dowling J. E. (1965) Foveal receptors of the monkey retina: fine structure Science 147, 57-59.

Enoch J. M. and Stiles W. S. (1961) The colour change of monochromatic light with retinal angle of incidence. Optica Acta 8, 329-358.

Green D. G. (1969) Sinusoidal flicker characteristics of the color-sensitive mechanisms of the eye. Vision Res. 9. $591-601$.

von Helmholtz H. (1896) Handhuch der Physiologischen Optik, 2nd ed, p. 369. L. Voss, Hamburg and Leipzig.

Mellerio J. (1971) Light absorption and scatter in the human lens. Vision Res. 11, 129-141.

Polyak S. L. (1941) The Retima. The University of Chicago Press, Chicago. Illinois.

Rushton W. A. H. (1963) Cone pigment kinetics in a protanope. J. Physiol. 168, 374-388.

Rushton W. A. H. (1965) Cone pigment kinetics in the deuteranope. $J$. Physiol. 176, 38 45.

Said F. S. and Weale R. A. (1959) The variation with age of the spectral transmissivity of the human crystalline lens. Gerentologia 3, 213-231.

Snyder A. W. (1969a) Asymptotic expressions for eigenfunction and eigenvalues of dielectric or optical waveguides. IEEE Trans. Microwate Theory Techn. MTT-17. $1130-1138$

Snyder A. W. (1969b) Excitation and scattering of modes on a dielectric or optical fiber. IEEE Trans. Microwave Theory Tech. MTT-17. 1134-1144.

Snyder A. W. and Pask C. (1973) The Stiles-Crawford effect- explanation and consequences. Vision Res. 13. $1115 \cdots 1137$.

Stiles W. S. (1937) The luminous efficiency of monochromatic rays entering the eye pupil at different points and a new colour effect. Proc. R. Soc. B123, 90-118.

Stiles W. S. (1939) The directional sensitivity of the retina and spectral sensitivities of the rods and cones. Proc, R. Soc. B127, 64-105.

Walraven P. L. and Bouman M. A. (1960) Relation between directional sensitivity and spectral response curves in human cone vision. J. opt. Soc. Am. $\mathbf{5 0}$. $780-784$.

Wyngaard M. A., Bouman M. A. and Budding F. (1974) The Stiles-Crawford colour change. Vision Res. 14. 951-957.

Wyszecki G. and Stiles W. S. (1967) Color Science. In Comcepts and Methods, Quantitative Data and Formulas. Wiley, New York.

\section{A. Self-screening hypothesis}

\section{APPENDIX}

For excitation, a critical amount of light has to be absorbed by the photopigment in the outer segment. One assumes that this amount does not depend upon where 
the pigment is located in the cone outer segment. When light is incident obliquely part of it passes into the surrounding tissue. which effectively shortens the length of the cone. Shortening the pathway through the pigment of the outer segment corresponds to narrowing the spectral absorption curve. Walraven and Bouman (1960) assume that there is a wavelength independent loss to the surrounding tissue which increases with increasing obliquity and is zero for normal incidence. For the case of normal incidence the fraction of light that is absorbed in the photopigment is

$$
I_{\mathrm{abs}}=I_{r_{m}}(\lambda)\left(1-\mathrm{e}^{-\alpha(\lambda) c l}\right)
$$

where

$I_{r_{m}}(\lambda)=$ The intensity (photons $\sec ^{-1} \mathrm{deg}^{-2}$ of yisual angle) of monochromatic light needed to reach a fixed criteria (just detectable $30 \mathrm{~Hz}$ flicker) at a pupil entry point $r_{m}$, (in $\mathrm{mm}$ ) from the geometric center to the point of entry of light into the observer's entrance pupil resulting in a maximum luminous efficiency.

$x(\lambda)=$ The absorption coefficiency of the photosensitive pigment in the cone outer segment in $\mathrm{cm}^{2}$ per chromophore.

$c=$ The concentration of photosensitive pigment in the cone outer segments in chromophores $\mathrm{cm}^{-3}$

$l=$ The length of the cone outer segments in $\mathrm{cm}$.

For oblique incidence the fraction of incident light absorbed is given by

$$
I_{\mathrm{abs}}=I_{r}(\lambda)\left(\frac{x(\lambda) c l}{\alpha(\lambda) c l+\xi l}\right)\left(1-\mathrm{e}^{-(x \mid \lambda) c+\xi t)}\right.
$$

where

$I_{r}(\lambda)=I_{r_{m}}(\lambda)$ at $r=r_{m} . r$ is the distance (in $\mathrm{mm}$ ) from the geometric center to the point of entry of light into the observer's entrance pupil.

$\xi=$ the fractional loss of light to the surround per unit length of cone outer segment. $\xi$ is assumed to be independent of wavelength. equal to zero for normal incidence and increasing with obliquity of the incident light beam.

The condition for equal visual effect leads to the definition of luminous efficiency

$$
\eta(\lambda)=\frac{I_{r_{m}}(\lambda)}{I_{r}(\lambda)}=\left(\frac{\alpha(\lambda) c l}{\alpha(\lambda) c l+\xi l}\right)\left(\frac{1-\mathrm{e}^{-\mid x(\lambda) c+\xi) l}}{1-\mathrm{e}^{-\alpha \mid \dot{\lambda}) c t}}\right) .
$$

Quantifying the Stiles-Crawford effect of the first kind by the empirical equation

$$
-\rho(\lambda)=\frac{1}{\left(r-r_{m}\right)^{2}} \log _{10} \eta(\lambda)
$$

and substituting equation (3) into (4)

$$
-\rho(\lambda)=\frac{1}{\left(r-r_{m}\right)^{2}} \log _{10}\left[\left(\frac{D(\lambda)+A}{D(\lambda)}\right)\left(\frac{1-10^{-D(\lambda)}}{1-10^{-(D(\lambda)+\lambda)}}\right)\right],
$$

in which

$$
\begin{aligned}
D(\hat{\lambda}) & =\alpha(\hat{\lambda}) c / \log _{10} \mathrm{e} \\
A & =\xi l \log _{10} \mathrm{e} .
\end{aligned}
$$

To solve equation (5) the action spectra for central pupil entry $\left(r_{m}\right)$ required to eliminate $30 \mathrm{~Hz}$ flicker for each observer after correction for losses in the lens and macular pigment was used. Thus, at any wavelength $\lambda$ the corresponding $D(\lambda)$ is derived from the action spectra curves illustrated in Figs 3 and 4 (by the broken lines) by using the equation

$$
\frac{I_{r_{m}}(\lambda)}{I_{r}(\lambda)}=\frac{1-10^{-D(\lambda)}}{1-10^{-D}}
$$

where $D=D(\lambda)$ at $\lambda_{\max }$.

Hence, given values for $D$ and $A$ the form of $\rho(\lambda)$ is uniquely determined. Enoch and Stiles' (1961) method for determining $D$ and $A$ were used. A computer program generated a family of curves $\rho$ as a function of $\lambda$.

\section{B. Waveguide model}

In the model of the Stiles-Crawford brightness effect of Snyder and Pask (1973) the variation of directional sensitivity with wavelength is attributed to the waveguide properties of the cones. The directional sensitivity of the receptor is quite independent of the spectral properties of the visual pigment in the cone outer segments; in this respect the theory differs sharply from the "self-screening" hypothesis presented above. The directional properties are determined by summing the total modal power which can be absorbed by the visual pigment in the cone outer segment. This is the modal power at the entrance to the taper (i.e. the interface of the outer and inner segments) which can be maintained by the outer segment structure. Al modes larger than $p=p^{\prime}$ and $q=q^{\prime}$ which are excited in the inner segment but are not capable of being sustained by the outer segment structure are assumed lost to the surrounding medium. The directional sensitivity of a single long- or medium-wave sensitive cone is evaluated by

$$
\rho(\lambda)=0.132 \log \frac{\sum_{1}^{p^{\prime}} \sum_{1}^{q^{\prime}} P_{p q}\left(\theta=6.875^{\circ}\right)}{\sum_{1}^{p^{\prime}} \sum_{1}^{q^{\prime}} P_{p q}(\theta=0)} .
$$

The modal power $P_{p q}$ was derived by Snyder (1969a, 1969b). It is

$$
P_{p q}=\frac{1}{Z}\left\{\frac{2 U_{q}}{V}\left[\frac{1}{U_{q}^{2}-\Delta^{2}}\right]\left[\frac{U_{q} J_{p}\left(W_{q}\right) J_{p-1}(\Delta)}{J_{p-1}\left(U_{q}\right)}-\Delta J_{p}(\Delta)\right]\right\}^{2}
$$

where

$$
\begin{aligned}
\Delta & =\pi d_{i} n_{i} \theta / \lambda \\
V & =\pi d_{i}\left[n_{i}^{2}-n_{s}^{2}\right]^{1 / 2} \lambda^{-1} \\
U_{q} & =U_{q}(\infty) \exp -V^{-1} \\
W_{q}^{2} & =V^{2}-U_{q}^{2} \\
Z & =K_{p}\left(W_{q}\right) K_{p-2}\left(W_{q}\right) K_{p-1}^{-2}\left(W_{q}\right) .
\end{aligned}
$$

The above equations use the following symbols:

$d_{i}=$ The diameter of the inner segment in microns.

$n_{i}=$ The index of refraction of the inner segment not including the taper.

$n_{s}=$ The index of refraction of the surrounding medium.

$\theta=$ The angle of incidence of the light on the inner segment in degrees

$=2.5\left(r-r_{m}\right)$

$J_{p-1}=$ The Bessel function of order $p-1$, (where $\left.p=1,2,3 \ldots p^{\prime}\right)$ is an integer representing the number of sign changes in the plane of the outer segment, inner segment interface of the electric modal vector as the azimuthal angle varies from 0 to $2 \pi$.

$K_{p-1}=$ The modified Hankel function of order $p-1$ (where $p=1,2,3 \ldots p^{\prime}$ ). 
$P_{p q}=$ The modal power of the $p q$ mode. $q=\left(1,2,3 \ldots q^{\prime}\right)$ is an integer where $q$ is the number of sign changes of the Bessel function dependence on the polar radius in the plane of the outer-inner segment interface.

$U_{q}(\infty)=$ The eigenvalue, i.e. the roots of the Bessel function of order $p-1 ; 2.405$ (for $p=1$ ): 3.832 (for $p=2$ ); 5.135 (for $p=3$ ).

To determine the limiting values of $p^{\prime}, q^{\prime}$ it should be appreciated that the number of modes propagated in the inner segment that will be absorbed by the visual pigment in the outer segment depends upon the wavelength (in vacuum) of the light. The lowest order of mode is propagated for all wavelengths, but higher order modes are limited to wavelengths $\lambda \leq \lambda$ by the equation

$$
\lambda^{\prime}=\pi d_{o}\left[n_{o}^{2}-n_{s}^{2}\right]^{1 / 2} U_{q}^{-1}(x)
$$

where

$n_{0}=$ The index of refraction of the cone outer segment including the taper.

$d_{o}=$ The diameter in microns of the cone outer segment.

For mode 2, $U_{q}(x)=2.405 ;$ for mode $3, U_{q}(x)=3.832$ and so on. If $n_{o}=1.43, d_{o}=1.00 \mu \mathrm{m}, n_{s}=1.34$, calculation shows that 3 modes are propagated for $\lambda=400 \mathrm{~nm}$ and $410 \mathrm{~nm}, 2$ modes for $\lambda$ between $410 \mathrm{~nm}$ and $650 \mathrm{~nm}$. but only the lowest order mode is propagated for $\lambda$ larger than $650 \mathrm{~nm}$.

These equations were used to generate a family of curves [ $\rho(\lambda)$ vs $\lambda]$ by varying the parameters $n_{i}, d_{i}$ and $d_{n}$ assuming $n_{o}=1.43$ and $n_{s}=1.34$. One member of this family of curves was selected for each dichromat and is presented (9) in Fig. 5. 